\title{
Carrier Lifetime Measurements in Long-Wave Infrared InAs/GaSb Superlattices Under Low Excitation Conditions
}

\author{
DING WANG,${ }^{1,2}$ DMITRI DONETSKY,${ }^{1}$ SEUNGYONG JUNG,${ }^{1}$ \\ and GREGORY BELENKY ${ }^{1}$ \\ 1.-Department of Electrical and Computer Engineering, Stony Brook University, Stony Brook, \\ NY 11794, USA. 2.—e-mail: wangding1985@gmail.com

\begin{abstract}
Minority carrier lifetime in long-wave infrared (LWIR) type II InAs/GaSb superlattices was studied using the optical modulation response (OMR) technique in wide ranges of excitation and temperature. The measured carrier lifetime was found to increase superexponentially with decreasing excitation power density below the level of $1 \mathrm{~mW} / \mathrm{cm}^{2}$ to $2 \mathrm{~mW} / \mathrm{cm}^{2}$. The phenomenon
\end{abstract} \\ was qualitatively explained by the presence of shallow trapping centers.
}

Key words: Carrier lifetime, long-wave infrared, superlattice

\section{INTRODUCTION}

Antimony-based type II strained-layer superlattices (T2-SLSs) are of great interest in the development of high-performance LWIR photodetectors and focal-plane arrays due to their bandgap tunability, material uniformity, and low Auger recombination. ${ }^{1-3}$ In the past several years, major efforts have been made to improve the performance of T2SLS photodetectors regarding growth, processing, and structural design. ${ }^{4-7}$ Current designs utilize a $p^{-}$-doped absorber and heterojunction barriers to reduce the generation-recombination and tunneling components of dark current. ${ }^{6}$ The performance of such photodetectors was reported to be limited by the Shockley-Read-Hall (SRH) carrier recombination process in the absorber. ${ }^{8}$

We previously reported the measurement of minority carrier lifetime in a LWIR T2-SLS structure with energy gap of $0.12 \mathrm{eV}$ at $77 \mathrm{~K}^{9}{ }^{9}$ The SRHlimited lifetime of $30 \mathrm{~ns}$ at $77 \mathrm{~K}$ was determined from the dependence of lifetime on excitation power using the OMR technique. Similar lifetime values were determined from diode $I-V$ characteristics ${ }^{10}$ and time-resolved photoluminescence (TRPL) decay measurements. ${ }^{11}$ In this work we present the results of minority carrier lifetime measurements performed at lower excess carrier densities (approximately from $10^{14} \mathrm{~cm}^{-3}$ to $10^{15} \mathrm{~cm}^{-3}$ ) in a wider temperature

(Received August 1, 2011; accepted July 25, 2012;

published online August 28, 2012) range (from $20 \mathrm{~K}$ to $250 \mathrm{~K}$ ). The results reveal interesting features of the mechanism of the minority carrier recombination process under low excitation conditions.

\section{EXPERIMENTAL PROCEDURES}

The SLS structure was grown by molecular beam epitaxy on a low-doped $p$-type GaSb substrate. The structure consists of a $1.8-\mu \mathrm{m}$-thick InAs/GaSb SLS layer (400 periods) enclosed within thin AlSbAs carrier confinement layers. A thin GaSb cap layer was grown on top of the structure to prevent oxidation. The SLS absorber had 13 monolayers (ML) of undoped InAs and $7 \mathrm{ML}$ of beryllium-doped GaSb. The target background hole concentration at $77 \mathrm{~K}$ was $1 \times 10^{16} \mathrm{~cm}^{-3}$. The structure exhibited a photoluminescence (PL) emission peak at $0.16 \mathrm{eV}$ at $77 \mathrm{~K}$

The minority carrier lifetime was measured by the OMR technique. ${ }^{9,12}$ Optical excitation was generated by a directly modulated $1.5-\mu \mathrm{m}$ fiber-coupled laser diode. The excitation beam was converged by a $\mathrm{CaF}_{2}$ lens; the beam width at half-maximum was $380 \mu \mathrm{m}$ on the specimen surface. We used a reflective objective to collect the PL within a solid angle of about 1.8 steradians. The PL was converged by a ZnSe lens onto a 250 - $\mu$ m-diameter liquid-nitrogencooled mercury cadmium telluride (MCT) photodetector. The modulated PL signal was selected and amplified by a Stanford Research radiofrequency (RF) lock-in amplifier (SR844). The narrow-band 
amplification of the sine-wave signal by the lockin amplifier improved the signal-to-noise ratio, allowing for the reduction of the optical excitation.

Two parameters were extracted from the fit of the PL frequency response to the dependence $\mathrm{PL}_{\omega} /\left[1+(\omega \tau)^{2}\right]^{0.5}$ : the carrier lifetime $\tau$, and the amplitude of the modulated PL signal in the low-frequency limit $(\omega \ll 1 / \tau), \mathrm{PL}_{\omega}$. When the modulation amplitude of the excitation is small compared with the steady-state excitation, the derivative of the steady-state PL intensity $\mathrm{PL}_{0}$ with respect to the steady-state carrier generation rate $G_{0}$ can be replaced by the ratio of the modulation amplitudes $\mathrm{PL}_{\omega} / G_{1}$, where $G_{1}$ is the amplitude of the modulated excitation in terms of the carrier generation rate. Therefore, $\mathrm{PL}_{\omega}$ can be expressed as

$$
\mathrm{PL}_{\omega}=\frac{\mathrm{dPL}_{0}}{\mathrm{~d} G_{0}} G_{1} \quad\left(\omega \ll 1 / \tau, G_{1} \ll G_{0}\right) .
$$

$G_{0}$ and $G_{1}$ were calculated from the excitation power and area assuming complete absorption of the optical excitation in the superlattice layer. The reflection of $35 \%$ of the excitation power at the interface between the sample and air was taken into account. The excitation power was controlled by either the laser operating current or the optical attenuation using a variable metallic neutral density filter.

\section{RESULTS AND DISCUSSION}

Figure 1a presents the PL frequency response data measured at $T=20 \mathrm{~K}$ for a series of excitation levels. The lifetime value derived from fitting was $\tau=26 \mathrm{~ns}$ at excitation of $13.6 \mathrm{~W} / \mathrm{cm}^{2}$, corresponding to excess carrier concentration $\Delta n=6 \times 10^{15} \mathrm{~cm}^{-3}$ or excess carrier generation rate $G_{0}=2.3 \times 10^{23} \mathrm{~cm}^{-3} \mathrm{~s}^{-1}$. A rapid decrease of the reciprocal carrier lifetime $1 / \tau$ towards low excitation was observed, as shown in Fig. 1b. A lifetime of $\tau=58 \mathrm{~ns}$ was obtained from fitting at excitation of $0.23 \mathrm{~W} / \mathrm{cm}^{2}$. The phenomenon
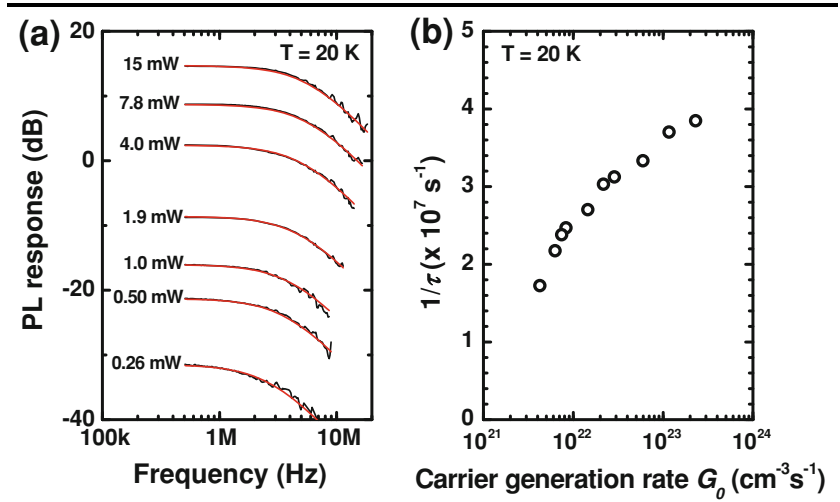

Fig. 1. (a) $\mathrm{PL}$ frequency response data at $20 \mathrm{~K}$ at various steadystate excitation powers from $0.26 \mathrm{~mW}$ to $15 \mathrm{~mW}$. Each response curve was fitted to the function $\mathrm{PL}_{\omega} /\left[1+(\omega \tau)^{2}\right]^{0.5}$. The excitation area was $1.1 \times 10^{-3} \mathrm{~cm}^{2}$. (b) Dependence of reciprocal carrier lifetime on the excess carrier generation rate at $20 \mathrm{~K}$. can be possibly associated with a shallow-traprelated recombination process, as discussed below.

In the steady-state condition, the generation and recombination rates of the excess carriers are equal:

$$
G_{0}=\frac{\Delta n}{\tau_{\mathrm{r}}},
$$

where $\tau_{\mathrm{r}}$ is the recombination lifetime of excess carriers. Previous results have shown that, in InAs/ GaSb superlattices, the SRH process dominates the recombination of excess carriers for a moderate level of excitation $\left(\Delta n=10^{15} \approx 10^{16} \mathrm{~cm}^{-3}\right){ }^{12,13}$ The measured values of $\tau_{\mathrm{r}}$ were typically in the range from $20 \mathrm{~ns}$ to $30 \mathrm{~ns}$, as shown in Fig. 2 (right $y$-axis) at $G_{0}>3 \times 10^{22} \mathrm{~cm}^{-3} \mathrm{~s}^{-1}$ (or $\Delta n>1 \times 10^{15} \mathrm{~cm}^{-3}$ ).

The steady-state minority excess carriers have a probability to recombine radiatively and generate PL. The steady-state PL intensity in a $p$-type material can be written as

$$
\mathrm{PL}_{0}=B^{\prime} \Delta n\left(\Delta n+p_{0}\right),
$$

where $B^{\prime}$ is the radiative recombination coefficient taking into account the collection efficiency of the detection system; $p_{0}$ is the background hole concentration at equilibrium. The parameter $\left(\mathrm{PL}_{\omega} / G_{1}\right)$ can be calculated from the derivative of $P L_{0}$ with respect to $G_{0}$, combining Eqs. $1-3$. Since $\tau_{\mathrm{r}}$ is a slow function of $G_{0}$ for $G_{0}>3 \times 10^{22} \mathrm{~cm}^{-3} \mathrm{~s}^{-1}$, the derivative of $\tau_{\mathrm{r}}$ is omitted and the expression can be simplified as

$$
\begin{aligned}
\frac{\mathrm{PL}_{\omega}}{G_{1}}= & \frac{\mathrm{dPL}_{0}}{\mathrm{~d} G_{0}} \approx B\left(p_{0}+2 G_{0} \tau_{\mathrm{r}}\right) \tau_{\mathrm{r}} \\
& \left(\omega \ll 1 / \tau, G_{1} \ll G_{0}, \mathrm{~d} \tau_{r} / \mathrm{d} G_{0} \approx 0\right) .
\end{aligned}
$$

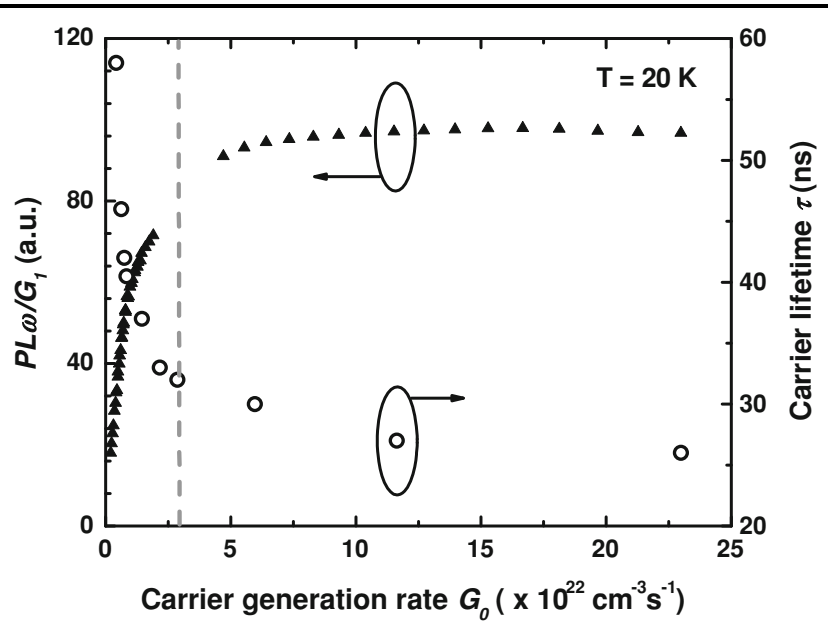

Fig. 2. Plot of $\left(\mathrm{PL}_{\omega} / G_{1}\right)$ as a function of the steady-state generation rate of excess carriers $G_{0}$ at $20 \mathrm{~K}$ (triangles, left $y$-axis). $\mathrm{PL}_{\omega}$ was measured at a low modulation frequency at $f=100 \mathrm{kHz}$; $G_{1}$ was set below $5 \%$ of $G_{0}$ during the measurement to validate Eq. 1 . The right $y$-axis shows the dependence of carrier lifetime on the excess carrier generation rate $G_{0}$ at $20 \mathrm{~K}$ (circles). The grey dashed line indicates $G_{0}=3 \times 10^{22} \mathrm{~cm}^{-3} \mathrm{~s}^{-1}$. 
Figure 2 (left $y$-axis) shows the measured $\left(\mathrm{PL}_{\omega} / G_{1}\right)$ as a function of $G_{0}$. For $G_{0}>3 \times 10^{22} \mathrm{~cm}^{-3} \mathrm{~s}^{-1},\left(\mathrm{PL}_{\omega} / G_{1}\right)$ was nearly constant, probably due to a relatively large background carrier concentration $\left(p_{0} » 2 G_{0} \tau_{\mathrm{r}}\right.$ ). Thus $\left(\mathrm{PL}_{\omega} / G_{1}\right)$ approximates to a linear function proportional to the recombination lifetime $\tau_{\mathrm{r}}$, especially at low excitations. However, when the excitation was decreased to the level of $G_{0}<3 \times 10^{22} \mathrm{~cm}^{-3} \mathrm{~s}^{-1}$, as shown in Fig. 2, $\left(\mathrm{PL}_{\omega} / G_{1}\right)$ dropped rapidly while the measured lifetime increased superexponentially. This discrepancy can be possibly attributed to the presence of shallow traps.

Shallow-trap-based models have commonly been used to explain the abnormally high effective lifetimes derived by photoconductance measurements under low injection in $\mathrm{Si}^{13,14}$ Trapping of minority carriers suppresses the recombination of excess carriers through SRH centers and results in a larger photoconductance and, therefore, an increased effective lifetime. The process may have a similar impact on the carrier lifetime measured by optical techniques. ${ }^{15}$ In the condition of low carrier injection, i.e., close to the density of shallow traps, a considerable portion of the steady-state excess electrons in $p$-type materials can be captured by the shallow traps. The captured electrons can escape from the traps to the conduction band at a slower rate, depending on the temperature, the energy levels of the traps, and other factors. This recycling process can decrease the overall rate of electron recombination through deep centers and could be the reason for the observed increase of the measured minority carrier lifetime. Statistically, since a considerable portion of the excess electrons is trapped, low PL intensity is also expected. Once the flux density of excitation photons is increased to a certain level, most of the shallow traps are occupied by electrons, so the measured lifetime will reflect the actual recombination rate of the minority electrons.

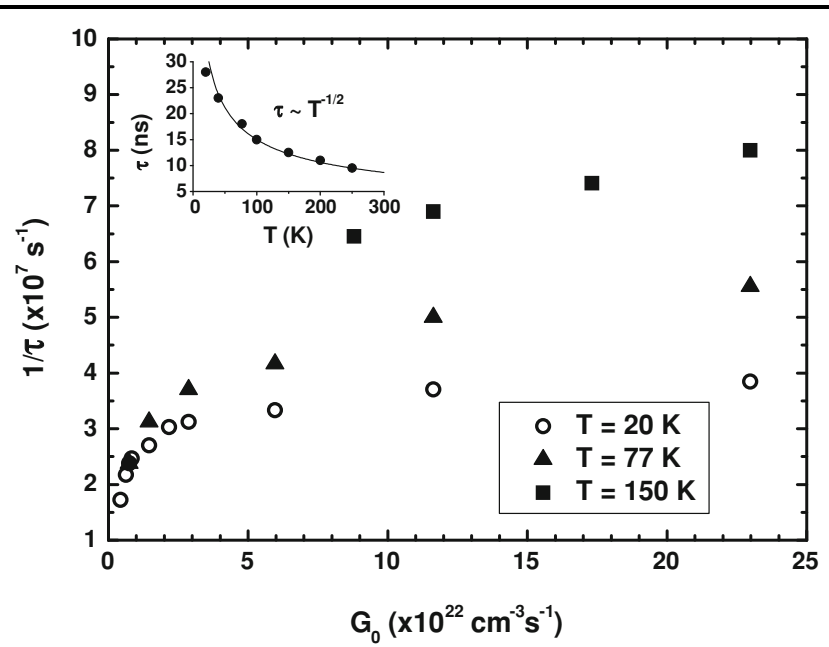

Fig. 3. Dependence of reciprocal carrier lifetime on excess carrier generation rate $G_{0}$ at $T=20 \mathrm{~K}$ (circles), $77 \mathrm{~K}$ (triangles), and $150 \mathrm{~K}$ (squares). The inset plots the minority carrier lifetime measured at $G_{0}=2.3 \times 10^{23} \mathrm{~cm}^{-3} \mathrm{~s}^{-1}$ as a function of temperature (dots). The line is the fit of the function $\tau \sim T^{-1 / 2}$.
The signal-to-noise ratio achieved in this work allowed carrier lifetime measurements in a wide temperature range up to $250 \mathrm{~K}$. Figure 3 presents the dependence of reciprocal carrier lifetime on excess carrier generation rate at $T=20 \mathrm{~K}, 77 \mathrm{~K}$, and $150 \mathrm{~K}$. A sharp decrease of $1 / \tau$ was apparent up to $77 \mathrm{~K}$. Determination of the carrier lifetime at low excitation was limited by PL quenching at elevated temperatures. The dependence of measured carrier lifetime $\tau$ on temperature followed $T^{-1 / 2}$ up to $250 \mathrm{~K}$ in the measured range of excitation, which can be attributed to the change of mean thermal velocity of excess carriers. The inset of Fig. 3 shows the carrier lifetime as a function of temperature measured at $G_{0}=2.3 \times 10^{23} \mathrm{~cm}^{-3} \mathrm{~s}^{-1}$.

\section{CONCLUSIONS}

We have presented the results of frequency-domain carrier lifetime measurements in $p$-doped LWIR T2-SLS under low excitation conditions. The lifetime value of $58 \mathrm{~ns}$ was obtained at the low excitation level $G_{0}=3 \times 10^{21} \mathrm{~cm}^{-3} \mathrm{~s}^{-1}$. A superexponential increase of the measured minority carrier lifetime was found when the excitation level decreased below $1 \mathrm{~mW} / \mathrm{cm}^{2}$ to $2 \mathrm{~mW} / \mathrm{cm}^{2}$. A qualitative explanation based on capture of excess electrons by shallow traps was proposed. The carrier lifetime was found to fit $\tau \sim T^{-1 / 2}$ in the temperature range up to $250 \mathrm{~K}$.

\section{ACKNOWLEDGEMENTS}

The authors would like to thank Dr. Stefan P. Svensson for helpful discussions. The LWIR SLS structures were provided by US Army NVESD. This research was supported by US Army NVESD and the National Science Foundation (Grant DMR0710154).

\section{REFERENCES}

1. G.A. Sai-Halasz, R. Tsu, and L. Esaki, Appl. Phys. Lett. 30, 651 (1977).

2. M. Razeghi, D. Hoffman, B.M. Nguyen, P.Y. Delaunay, E.K.W. Huang, M.Z. Tidrow, and V. Nathan, Proc. IEEE 97, 1056 (2009).

3. E.R. Youngdale, J.R. Meyer, C.A. Hoffman, F.J. Bartoli, C.H. Grein, P.M. Young, H. Ehrenreich, R.H. Miles, and D.H. Chow, Appl. Phys. Lett. 64, 3160 (1994).

4. M. Walther, J. Schmitz, R. Rehm, S. Kopta, F. Fuchs, J. Flei $\beta$ ner, W. Cabanski, and J. Ziegler, J. Cryst. Growth 278, 156 (2005).

5. A. Khoshakhlagh, E. Plis, S. Meyers, Y.D. Sharma, L.R. Dawson, and S. Krishna, J. Cryst. Growth 311, 1901 (2009).

6. I. Vurgaftman, E.H. Aifer, C.L. Canedy, J.G. Tischler, J.R. Meyer, J.H. Warner, E.M. Jackson, G. Hildebrandt, and G.J. Sullivan, Appl. Phys. Lett. 89, 121114 (2006).

7. D.Z.Y. Ting, C.J. Hill, A. Soibel, S.A. Keo, J.M. Mumolo, J. Nguyen, and S.D. Gunapala, Appl. Phys. Lett. 95, 023508 (2009).

8. I. Vurgaftman, C.L. Canedy, E.M. Jackson, J.A. Nolde, C.A. Affouda, E.H. Aifer, J.R. Meyer, A. Hood, A.J. Evans, and W.T. Tennant, Opt. Eng. 50, 061007 (2011).

9. D. Donetsky, G. Belenky, S.P. Svensson, and S. Suchalkin, Appl. Phys. Lett. 97, 052108 (2010).

10. J. Pellegrino and R. DeWames, Proc. SPIE 7298, 72981U (2009). 
11. B.C. Connelly, G.D. Metcalfe, H. Shen, and M. Wraback, Appl. Phys. Lett. 97, 251117 (2010).

12. D. Donetsky, S.P. Svensson, L.E. Vorobjev, and G. Belenky, Appl. Phys. Lett. 95, 212104 (2009).

13. N.P. Harder, R. Gogolin, and R. Brendel, Appl. Phys. Lett. 97, $112111(2010)$
14. K.R. McIntosh, B.B. Paudyal, and D.H. Macdonald, J. Appl. Phys. 104, 084503 (2008).

15. S. Bandara, P. Maloney, N. Baril, J. Pellegrino, and M. Tidrow, Opt. Eng. 50, 061015 (2011). 